\title{
Subject-specific aortic wall shear stress estimations using semi-automatic segmentation
}

\author{
Johan Renner, Hossein Nadali Najafabadi, Daniel Modin, Toste Länne and M Karlsson
}

\section{Linköping University Post Print}

N.B.: When citing this work, cite the original article.

This is the authors' version of the following article:

Johan Renner, Hossein Nadali Najafabadi, Daniel Modin, Toste Länne and M Karlsson, Subject-specific aortic wall shear stress estimations using semi-automatic segmentation, 2012, Clinical Physiology and Functional Imaging, (32), 6, 481-491.

which has been published in final form at:

http://dx.doi.org/10.1111/j.1475-097X.2012.01146.x

Copyright: Wiley-Blackwell

http://eu.wiley.com/WileyCDA/Brand/id-35.html

Postprint available at: Linköping University Electronic Press

http://urn.kb.se/resolve?urn=urn:nbn:se:liu:diva-85079 


\title{
Subject Specific Aortic Wall Shear Stress Estimations using
}

\section{Semi-Automatic Segmentation}

\author{
J. Renner and H. Nadali Najafabadi and D. Modin \\ and T. Länne and M. Karlsson
}

\begin{abstract}
Atherosclerosis development is strongly believed to be influenced by hemodynamic forces such as wall shear stress (WSS). To estimate such an entity in-vivo in humans, image based computational fluid dynamics (CFD) is a useful tool. In this paper we use a combination of magnetic resonance imaging (MRI) and CFD to estimate WSS. In such method a number of steps are included. One important step is the iterpretation of images into 3D models, named segmentation. The choice of segmentation method can influence the resulting WSS distribution in the human aorta. This is studied by comparing WSS results gained from the use of two different segmentation approaches: manual and semi-automatic, where the manual approach is considered to be the reference method. The investigation is performed on a group of 8 healthy male volunteers.
\end{abstract}

The different segmentation methods give slightly different geometrical depictions of the human aorta (difference in the mean thoracic Aorta lumen diameter were $0.7 \%$ p 0.86 ). However, there is a very good agree-

J.Renner · H.Nadali Najafabadi · M. Karlsson

Department of Mechanical Engineering, Linköping University, Sweden,

E-mail: johan.renner@liu.se

D. Modin · T. Länne

Department of Medicine and Health Sciences, Linköping University, Sweden 
ment between the resulting WSS distribution for the two segmentation approaches. The small differences in

WSS between the methods increase in the late systole and early diastolic cardiac cycle time point indicating that the WSS is more sensitive to local geometric differences in these parts of the cardiac cycle (correlation coefficient is 0.96 at peak systole and 0.68 at early diastole). We can conclude that the results show that the semi-automatic segmentation method can be used in the future to estimate relevant aortic WSS.

\section{Introduction}

In the Western world, many people die of cardiovascular diseases e.g. myocardial infarction and stroke. One of the main reasons is atherosclerosis development in the cardiovascular system, which involves a complex process in the arterial wall not yet fully understood, [1]. The cardiovascular system is constantly subjected to biomechanical stimulation, generated by the flowing blood and it is widely recognized that differences in flow characteristics play a major role in the pathogenesis of vascular diseases. Early atherosclerosis lesions predominately form at specific arterial regions such as arterial branching and at the inner curvature of the aortic arch, in which the blood flow is complex (both in space and time) resulting in disturbed wall shear stress (WSS) distribution [2,24,25,23]. Atherosclerotic lesions can, e.g., be found in the human aorta [9]. Non-invasive imaging combined with computational fluid dynamics (CFD) is a powerful tool $[3,4,5,6,7]$ in order to estimate such hemodynamic forces.

The approach used in this paper has the capability to in-vivo estimate hemodynamic forces such as WSS inside a specific human aorta [8]. WSS distribution in the human aorta has earlier been studied $[5,11,12]$. However, these studies have certain limitations, such as: 1) only small aortic segments have been studied, 2) geometrically idealized/simplified models 
of the aortic arch have been used and 3) only a limited number of subjects investigated. To perform estimations of subject specific WSS, a wide range of research disciplines must be included; from image collection to advanced flow calculations. In order to gain knowledge regarding 3D arterial geometries, computed tomography (CT), magnetic resonance imaging (MRI) and ultrasound can in principle be used to collect the image data. Reconstructed geometries can then be integrated with CFD simulations to estimate biomechanical factors (e.g. WSS) in the healthy or diseased cardiovascular system. Manual, automated or semiautomated segmentation methods may be used in the interpretation of image data to define the lumen of the vessel and accordingly build 3D geometries. In this work, the images of the human aorta are acquired by the use of MRI modality. These images are processed using segmentation techniques that produce a three dimensional model of the arterial wall location. CFD is then used to calculate flow data in the human aorta and the result is subsequently processed to acquire the WSS distribution at the arterial wall. Furthermore, the influence of the segmentation method on the WSS has been the main focus of the investigation. Since the geometrical representation of the artery may influence WSS distribution [10, $13,14,8]$, it is of great interest to evaluate the effect of the geometrical interpretation method (segmentation) on the resulting WSS values. In studies performed by $[19,20]$, automated or semi-automated segmentation methods were used to reconstruct 3D geometries, but limited attention was given to the effects of segmentation method on the quantification of biomechanical factors. However, in another study by [22], 3D ultrasound data acquisition has utilized manual and semi-automated segmentation methods to produce 3D geometries of carotid bifurcation prepared for CFD analysis. The concludsion was that further work has to be performed on larger datasets to optimize and compare manual and semi-automated segmentation methods [22]. 
This paper aim is to analyze the sensitivity of the estimated WSS distribution depending on manual and semi-automatic segmentation methods applied to the human aorta in a group of 8 healthy volunteers. The analysis is to be performed on spatially detailed as well as time-resolved data. 


\section{Method}

The major steps of the method for estimating subject specific WSS in the human aorta have been described in [8], but are briefly mentioned here. Steps included in the process are image acquisition, segmentation, meshing, CFD setup, CFD simulation and CFD post-processing. Further, this section will also include the statistical approach to analyze the results.

Material

The ages of the 8 healthy male volunteers included in the study ranged from 21 to 26 years. All volunteers gave informed consent, and the study was approved by the regional Ethics Committee for Human Research at Linköping University, Sweden. The full data about the subjects is found in table 1 . Note the very small spread in both age $(23.5 \pm 1.3)$ and BMI $(21.8 \pm 1.4)$ of the volunteers. The volunteers were healthy with no history of cardiovascular disease.

Table 1 Age, weight, length, BMI and blood viscosity of the eight subjects.

\begin{tabular}{|c|c|c|c|c|c|}
\hline Subject & Age [years] & Weight $[\mathrm{kg}]$ & Height $[\mathrm{m}]$ & BMI $\left[\mathrm{kg} / \mathrm{m}^{2}\right]$ & Viscosity $[\mathrm{kg} / \mathrm{ms}]$ \\
\hline 1 & 24 & 68 & 1.79 & 21.2 & 0.0043 \\
\hline 2 & 23 & 69 & 1.81 & 21.1 & 0.0039 \\
\hline 3 & 21 & 67 & 1.82 & 20.2 & 0.0045 \\
\hline 4 & 23 & 73 & 1.86 & 21.1 & 0.0046 \\
\hline 5 & 24 & 72 & 1.87 & 20.6 & 0.0046 \\
\hline 6 & 26 & 71 & 1.78 & 22.4 & 0.0055 \\
\hline 7 & 23 & 83 & 1.84 & 24.5 & 0.0037 \\
\hline 8 & 24 & 75 & 1.79 & 23.4 & 0.0041 \\
\hline \hline & $23.5 \pm 1.3$ & $72 \pm 5$ & $1.82 \pm 0.03$ & $21.8 \pm 1.4$ & $0.0044 \pm 0.0005$ \\
\hline
\end{tabular}


The in-vivo measurement technique of MRI was used in order to obtain both geometrical as well as flow information. The eight healthy volunteers were scanned in a $1.5 \mathrm{~T}$ MRI scanner (Philips Achieva, Philips Medical Systems, Best, The Netherlands). Geometrical data of the whole aorta was acquired with a gadolinium-enhanced gradient-echo sequence. The final geometrical data was transferred into a resolution of $0.78 \times 0.78 \times 1.00 \mathrm{~mm}^{3}$. One component (main flow direction) velocities were measured in a plane perpendicular to the main flow direction in the ascending aorta distal to the aortic root. Velocity information was gained in a $10 \mathrm{~mm}$ thick slice-segment with an in-plane spatial resolution of $1.37 \times 1.37 \mathrm{~mm}^{2}$. The temporal resolution was approximately 40 time-frames in each cardiac cycle. More detailed information about the employed MRI strategy is found in [8].

\section{Segmentation}

Segmentation was performed with two methods: a manual and a semi-automatic approach. Both approaches were conducted on the same MRI image data material and by the same operator.

\section{Manual method}

In order to locate the aortic wall, the manual method was used by placing points manually in the lumen blood interface, see figure 1. At least 1000 points were placed manually at the aortic luminal surface on every image slice containing parts of the aorta with a density of ten points per $\mathrm{cm}$. This was done with the aid of in house-developed software in Matlab, that gave the operator a tool to place points at the luminal surface on the MRI slices and collect the coordinates all these points. In this manner, the investigator defined the geometry of the entire aorta. The produced point-cloud from all image slices (about 20000 points) was then 
used to describe the 3D geometry of the entire aortic luminal surface. This cloud of points was subsequently converted into a tessellation surface and smoothed in the CAD (Computer Aided Design) software Catia V5 (Dassault Systemes, Vélizy-Villacoublay Cedex, France) in order to get a usable geometrical description for the following steps in the WSS estimation method.
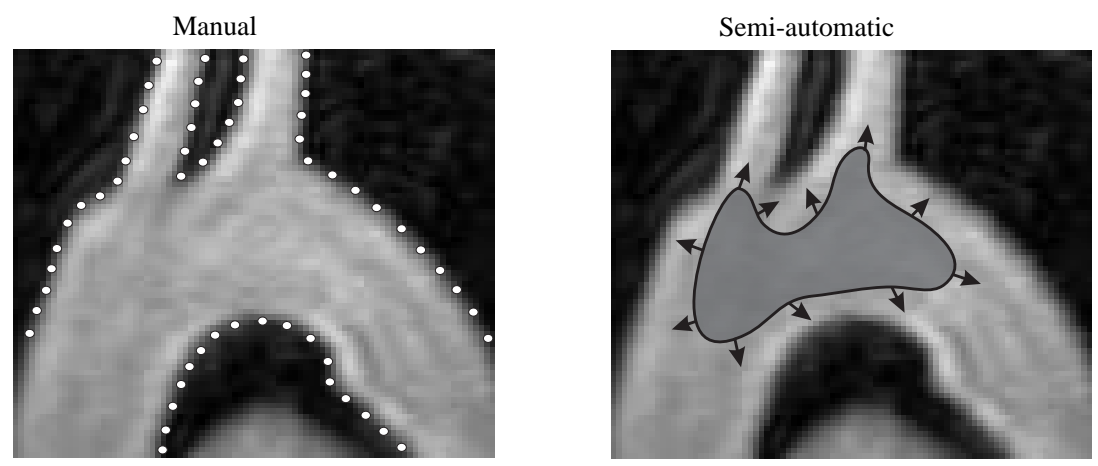

Fig. 1 Left: Cartoon image of manual segmentation, MRI slice of the aortic arch with manually placed points marking the interface between the luminal wall surface and the blood. Right: Cartoon image of semiautomatic segmentation with seed point/region that expands in 3D until it reaches the vessel wall location.

\section{Semi-automatic method}

The semi-automatic segmentation procedure uses a level set method, where a region is expanded out from one or several seed points [16]. The seed points are placed by the operator and expand automatically toward the aortic contours. The segmentation data was then smoothed with a $2 \mathrm{~mm}$ Gaussian smoothing filter with a spatial size of 19 pixels. Smoothing filter size (2mm) was selected based on tests on a wide range of settings, which aimed at smoothing inacurate rough surfaces, all the while minimizing oversmoothing. Approximate time for the segmentation process was 20 minutes per subject including the needed manual interactions, e.g., avoid including the pulmonary artery in the segmentation. The 
segmentation algorithm was implemented into the cardiac image analysis software package

Segment [15]. This software was also used to obtain velocity information from the MRI measurements in the ascending and descending aorta. The time resolved volume flow rate was acquired for further use in the CFD boundary conditions, which will be addressed later.

The resulting inlet and outlet areas of both segmentation methods is viewed in table 2 .

Table 2 Area of inlet and outlets in all subjects using manually and semi-automatic segmentation.

\begin{tabular}{|c|c|c|c|c|c|c|}
\hline \multirow[t]{2}{*}{ Subject } & \multirow[t]{2}{*}{ Segmentation } & \multicolumn{5}{|c|}{ Area $\left(\mathrm{cm}^{2}\right)$} \\
\hline & & Inlet & Outlet 1 & Outlet 2 & Outlet 3 & Outlet 4 \\
\hline \multirow{2}{*}{1} & Semi-Automatic & 6.46 & 1.41 & 0.41 & 0.97 & 2.98 \\
\hline & Manual & 6.00 & 1.35 & 0.49 & 0.87 & 2.72 \\
\hline \multirow{2}{*}{2} & Semi-Automatic & 4.60 & 0.69 & 0.28 & 0.58 & 3.46 \\
\hline & Manual & 5.38 & 0.98 & 0.29 & 0.67 & 3.73 \\
\hline \multirow{2}{*}{3} & Semi-Automatic & 7.27 & 1.08 & 0.40 & 0.65 & 2.64 \\
\hline & Manual & 7.49 & 1.21 & 0.33 & 0.63 & 2.80 \\
\hline \multirow{2}{*}{4} & Semi-Automatic & 4.40 & 0.92 & 0.41 & 0.63 & 2.22 \\
\hline & Manual & 5.01 & 1.11 & 0.53 & 0.86 & 2.37 \\
\hline \multirow{2}{*}{5} & Semi-Automatic & 5.90 & 1.11 & 0.44 & 0.52 & 2.64 \\
\hline & Manual & 6.18 & 1.30 & 0.48 & 0.56 & 2.76 \\
\hline \multirow{2}{*}{6} & Semi-Automatic & 5.07 & 1.02 & 0.50 & 0.82 & 2.29 \\
\hline & Manual & 5.30 & 1.15 & 0.51 & 0.96 & 2.44 \\
\hline \multirow{2}{*}{7} & Semi-Automatic & 5.21 & 1.28 & 0.54 & 0.97 & 3.66 \\
\hline & Manual & 5.42 & 1.40 & 0.49 & 1.03 & 3.96 \\
\hline \multirow{2}{*}{8} & Semi-Automatic & 7.04 & 1.35 & 0.53 & 0.62 & 2.41 \\
\hline & Manual & 7.42 & 1.52 & 0.54 & 0.65 & 2.58 \\
\hline
\end{tabular}


Fluid Model

The surface descriptions reconstructed from MRI acquisition were subject to mesh generation and quality check using ICEM 12.0.1 (Ansys, Inc., Canonsburg, Pennsylvania, USA). To generate a mesh from the delineated surfaces, some geometric corrections were acquired. This was done by cropping the surface at the inlet and at the outlets, see the differences between the left and right part of figure 2 . This renovation was necessary due to the smoothing operations in the segmentation. The areas for inflow and outflow were prepared to introduce boundary conditions. These surface edges had to be sharp so there is a distinct difference between the wall surface and the inlet and outlet surfaces. 
The corrections were also used to obtain approximately the same geometrical section of the aorta for both manual and semi-automatic segmentation methods for all subjects. This results in consistency between the two different models of the same subject as well as consistency among the various subjects. The distance in which all inlet/outlets were cut, was measured from a topologically well defined point $(\Theta)$, which was unique in for all subjects. This point $\Theta$ was located in curvature indicating subclavian artery, see figure 2. Although the effort in placing this point manually introduced the errors in size of $\pm 1 \mathrm{~mm}$, this discrepancy is considered small and neglectable with respect to the final results.

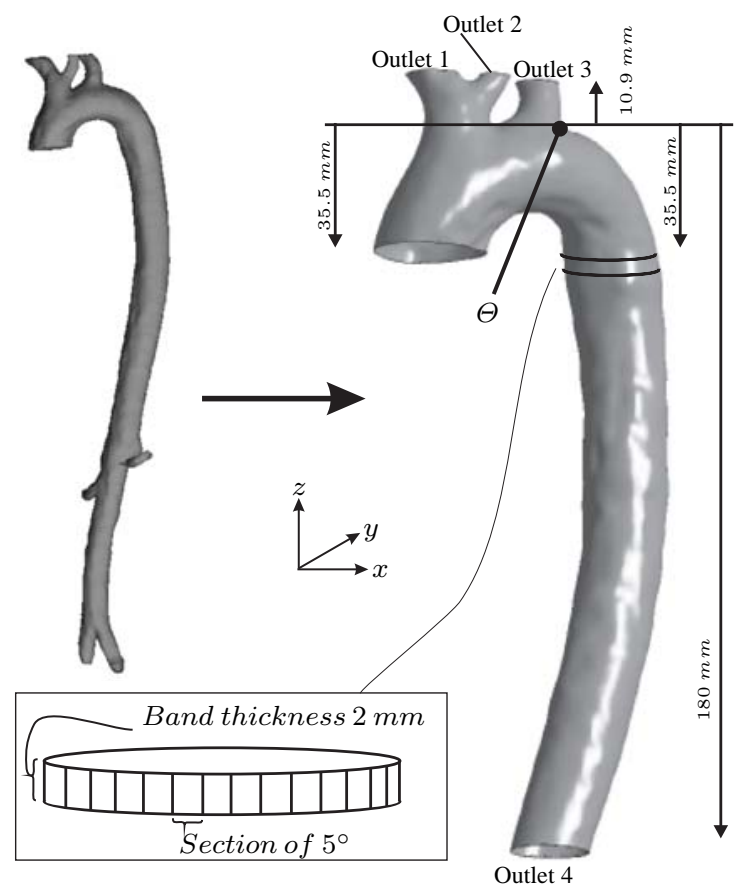

Fig. 2 Left: Geometrical description of the human aorta from segmentation, the uncropped geometry. Right: Geometrical description after cropping with the location of the comparison section marked. The $\Theta$ represents the point where all distance measurements originate. This point is located on the outer curvature of the aortic arch at the end of the subclavian artery branching. 
Three specific distances, all measured from point $\Theta$, have been used to consequently define inlet and outlet locations, see figure 2. The distances chosen for this purpose were calculated based on the fraction of the average inlet diameter of all subjects $\left(\bar{D}_{\text {inlet }}=27.3 \pm 2.3 \mathrm{~mm}\right)$. The distances in figure 2 were defined as multiplications of $\bar{D}_{\text {inlet }}$ in head/feet direction, by fraction of 1.3 for inlet, 0.4 for outlets $1,2,3$ and a fraction 6.6 for outlet 4 . These fractions were chosen in order to avoid any outflow boundary condition effects in the analyzed region and to be consistent regarding the aortic geometries used in the CFD simulations.

The mesh creation step within a CFD analysis constitutes one of the most important steps. The high importance of mesh generation is due to its effect in determination of the numerical solutions' accuracy. In general, the size, type and quality of the generated mesh can compromise the convergence and stability of the computational solution. Consequently, a well-constructed mesh deserves great attention in a CFD simulation. Therefore the computation mesh strategy has been developed by conducting extensive mesh tests regarding quality as well as mesh independence in the solutions. For this purpose, one segmentation type (semi-automatic) for a randomly selected subject was identified, and several simulations were conducted toward mesh independence for both velocity and WSS quantities. Mesh independence was reached for meshes with Tri/Tetra elements and six prism layers near the wall, with an approximate total size of $2.7 \cdot 10^{6}$ elements (including both Tri/Tetra and prism elements). The prism layers were added to resolve the region near the wall to acquire an accurate prediction of boundary layer quantities such as WSS.

The mesh quality is crucial to achieve convergence, stability and accuracy of the final computational result. The following mesh quality criteria were checked: determinant, skewness and general quality. Each of these factors depends on several other factors e.g., size, type, 
and location of the cells. In all cases, the general quality of the mesh was improved to a certain satisfactory level, with the general quality being $>0.3$ for all meshes. Solver settings for CFD simulations were performed utilizing the commercial software FLUENT version 12.0.1 (Fluent Inc., Lebanon, New Hampshire, USA). The simulations were carried out on the Linux cluster at the National Supercomputer Center (NSC, Linköping University, Sweden). The software uses finite-volume method for discretization of the equations. The mathematical model for blood flow utilizes three-dimensional, time-dependent Navier-Stokes equations for an incompressible and Newtonian fluid.

$$
\begin{aligned}
& \nabla \cdot \mathbf{V}=0 \\
& \frac{\partial(\rho \mathbf{V})}{\partial t}+\nabla \cdot(\rho \mathbf{V} \mathbf{V})=-\nabla p+\mu \nabla^{2} \mathbf{V}
\end{aligned}
$$

The governing equations include continuity and momentum equations, where $\boldsymbol{V}$ is the velocity vector, $p$ the pressure, $\rho$ the density, and $\mu$ the dynamic viscosity, see equations 1 and 2 . The blood was assumed incompressible, with a density of $1060 \mathrm{~kg} / \mathrm{m}^{3}$ and Newtonian with constant dynamic viscosity $\mu$ measured for each subject, see table 1 (measurement details are found in [8]).

Moreover, the total volume flow rate measured with MRI in cross sections placed in the ascending and descending aorta has been used for specification of the inflow/outflow boundary conditions. Volume flow rate in the ascending aorta was utilized to provide a fully developed laminar parabolic velocity profile in the inlet boundary condition based on equationa 3 and 4 . This velocity profile was chosen in order to get consistent volume flow rate conditions for the slightly varying geometries gained from the different segmentation approaches. The inflow boundary condition uses the measured one component velocity information and in the real ascending aorta flow situation there is also secondary velocity components normally forming a spiral like flow and this situation is not modeled. But the study aims at compar- 
ing segmentation methods and the exclusion of the secondary velocities is assumed to have neglectable influence on the main results.

$$
\begin{gathered}
V(x, y)=V_{\max }\left(1-\left(\frac{x^{2}+y^{2}}{r^{2}}\right)\right) \\
V_{\max }=\frac{\dot{V}_{\text {inlet }}}{A_{\text {inlet }}} \cdot 2
\end{gathered}
$$

Where inlet flow $\dot{V}$ was based on subject specific measurement of the blood velocity and $x$ and $y$ are the corresponding coordinate values of the inlet face (figure 2) and $r$ is the radius. In order to obtain the weighted outflow for the outlets, the flow weight fraction $\Psi$ for each outlet was derived. The outlet fractions were based on the flow measurements in the ascending and descending aorta where the fraction in outlet 4 was directly gained from the measured flow, see equation 5. In outlets 1-3 the fraction $\Psi$ was based on the remaining flow rate and the outlet boundary area according to equation 6 , see table 3 . Table 3 presents the complete list of outflow fractions used.

$$
\begin{gathered}
\Psi_{\text {outlet }(4)}=\frac{\dot{V}_{\text {outlet }(4)}}{\dot{V}_{\text {inlet }}} \\
\Psi_{\text {outlet }(i)}=\left(1-\frac{\dot{V}_{\text {outlet }(4)}}{\dot{V}_{\text {inlet }}}\right)\left(\frac{A_{\text {outlet }(i)}}{\sum_{i=1}^{3} A_{\text {outlet }(i)}}\right) \quad i=1,2,3
\end{gathered}
$$

The use of these area weighted outflow fractions in the aortic arch brances is as well a simplification of the real flow situation where the distal resistence to these outflow sites influences the flow fractions. Thus the approach used is belived to be sufficient enough to be able to compare the different segmentation methods. Especially as the comparison site (to be described later) is located in the descending aorta and the flow fraction of the outflow distal to this site is measured. The aortic wall was assumed to be a rigid wall with a no 
Table 3 Flow fractions for the different outlets based on the areas in table 2.

\begin{tabular}{|c|c|c|c|c|c|}
\hline \multirow[t]{2}{*}{ Subject } & \multirow[t]{2}{*}{ Segmentation } & \multicolumn{4}{|c|}{ Flow fraction $\Psi(\%)$} \\
\hline & & Outlet 1 & Outlet 2 & Outlet 3 & Outlet 4 \\
\hline \multirow{2}{*}{1} & Semi-Automatic & 15 & 9 & 15 & 61 \\
\hline & Manual & 19 & 7 & 13 & 61 \\
\hline \multirow{2}{*}{2} & Semi-Automatic & 16 & 6 & 13 & 65 \\
\hline & Manual & 18 & 5 & 12 & 65 \\
\hline \multirow{2}{*}{3} & Semi-Automatic & 23 & 9 & 14 & 54 \\
\hline & Manual & 26 & 7 & 13 & 54 \\
\hline \multirow{2}{*}{4} & Semi-Automatic & 16 & 7 & 11 & 66 \\
\hline & Manual & 15 & 7 & 12 & 66 \\
\hline \multirow{2}{*}{5} & Semi-Automatic & 21 & 8 & 10 & 61 \\
\hline & Manual & 22 & 8 & 9 & 61 \\
\hline \multirow{2}{*}{6} & Semi-Automatic & 13 & 6 & 10 & 71 \\
\hline & Manual & 13 & 5 & 11 & 71 \\
\hline \multirow{2}{*}{7} & Semi-Automatic & 15 & 6 & 12 & 67 \\
\hline & Manual & 16 & 5 & 12 & 67 \\
\hline \multirow{2}{*}{8} & Semi-Automatic & 17 & 6 & 8 & 69 \\
\hline & Manual & 17 & 6 & 8 & 69 \\
\hline
\end{tabular}

slip boundary condition. The required time step size for transient simulations was evaluated based on Courant-Friedrichs-Lewy (CFL) condition. For all simulations the time step was set to $1.84 \mathrm{~ms}$ in order to satisfy the CFL condition. In addition, second order discretization (Green-Gauss Node based) was used for higher accuracy in the numerical simulation. The numerical scheme chosen for pressure velocity coupling was the SIMPLE algorithm.

To be able to compare WSS results, specific locations were defined for detailed analysis by introducing a circumferential band at the descending aorta. The aim of this study is to compare WSS using manual and semi-automatic segmentation methods, we introduced 
bands with the thickness of $0.002 \mathrm{~m}$, see figure 2 . The comparison band is located in the descending aorta at the same head/feet coordinate as the inlet, i.e. $1.28 \cdot \bar{D}_{\text {inlet }}$ (average inlet diameter) in feet direction from point $\Theta$. WSS was averaged over every $5^{\circ}$ segment of the band to obtain the WSS circumferential details. Tests were made with different band thickness and section sizes to avoid any influence the choosen post-processing method may have on the results. An optimum was found for the chosen settings described above. For less detailed analysis the whole band averaged WSS has been used. Regarding WSS extraction the included capabilities in FLUENT were used. This includes flow properties adjacent to the wall/fluid boundary for prediction of shear stresses, obviously this holds for no-slip wall conditions.

$$
W S S=\left.\mu \cdot \frac{\partial v_{t}}{\partial n}\right|_{w a l l}
$$

Since the flow is considered to be laminar, the wall shear stress is calculated by multiplying the viscosity $\mu$ and the velocity $\left(v_{t}\right)$ (component tangential to the wall at the wall) gradient normal $(n)$ to the wall according to equation 7.

\section{Statistics}

In order to statistically compare WSS results from the two segmentation methods the Pearson product-moment correlation coefficient $r$ has been utilized according to equation 8 [18].

$$
r=\frac{\sum\left(X_{i}-\bar{X}\right)\left(Y_{i}-\bar{Y}\right)}{\left[\sum\left(X_{i}-\bar{X}\right)^{2}\left(Y_{i}-\bar{Y}\right)^{2}\right]^{1 / 2}}
$$

where $X$ and $Y$ correspond to WSS magnitude values from manual segmentation and semiautomatic segmentation, respectively and $\bar{X}$ and $\bar{Y}$ are the mean WSS magnitudes. The correlation coefficient was calculated on both detailed data $\left(5^{\circ}\right.$ segments $)$ as well as averaged data (circumferential average) to enlighten different views of the results. To fully describe 
the different aspects of the data, correlation coefficients are not enough according to Bland and Altman [17] who distinguish the level of relation and agreement between two methods, particularly when using a correlation coefficient. This is best understood by viewing two different types of plots. First, plot the result of one method against the result of another and second, plot the difference between the methods against their means. These plotting approaches will be used in this work to complement the use of correlation coefficients.

\section{Results}

To show geometrical similarities between the two segmentation methods, an overview of the cross-sectional areas at inlets and outlets for the whole group is presented in figure 3 (based on table 2 data). Mean thoracic aorta (TA) lumen diameter (LD) for the semi-automatic seg-

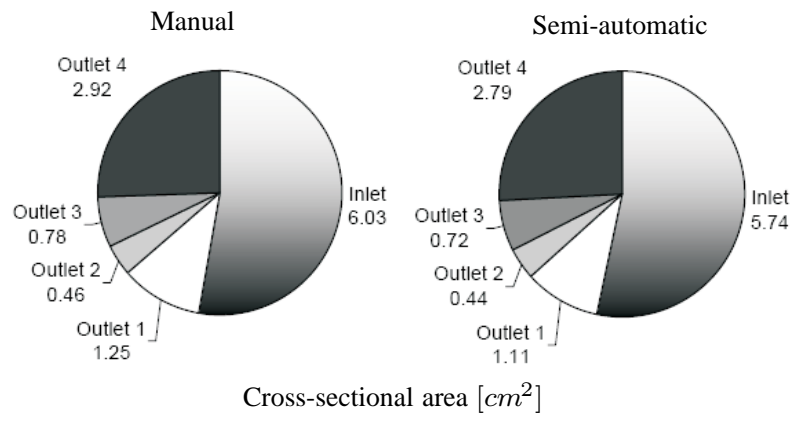

Fig. 3 Inlet and outlet cross-sectional average areas for manual (left) and semi-automatic (right) segmentation methods (based on table 2 data).

mentation was $19.3 \pm 1.4 \mathrm{~mm}$ and for the manual method $19.2 \pm 1.4 \mathrm{~mm}$. The difference in mean LD for the TA segment was $0.7 \%$. No significant differences between the segmentation methods in the TA lumen diameter were found, with $\mathrm{p}<0.86$. The intra- and interobserver variability for the semi-automatic segementations for the LD are viewed in figure 4 . No further variability analysis regarding WSS is performed due to the low variability in the LD. 
According to [28], since the flow variability is reported low, the WSS variability is expected to be low as well because the employed CFD protocol is consistent for all subjects. It should only be influenced by the 3D geometry and the flow as input data to the CFD protocol.
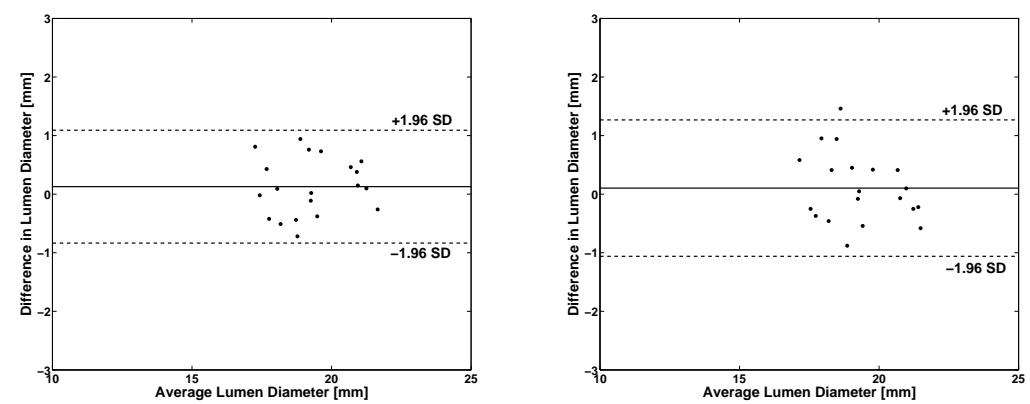

Fig. 4 Left: Intraobserver variability obtained for the semi-automatic thoracic aortic lumen diameter. Right: Interobserver variability obtained for the semi-automatic thoracic aortic lumen diameter. Lumen diameter is defined as the diameter in the anterior-posterior direction.

There are different ways of viewing flow-field data of a time resolved CFD simulation. Indeed, one can visualize static images during a specific time point or time-resolved video. For the purpose of this study, four time points during the cardiac cycle have been selected for comparison of the WSS variation between two segmentation methods. The chosen time points in the cardiac cycle were: systolic acceleration, peak systole, systolic deceleration and early diastole. Altough massive amounts of flow data were generated within this research, only flow data at the desribed locations (both in space and time) will be illustrated.

Visualization of contours for a particular quantity, e.g. WSS, can give an overall impression of the quantitative distribution. Circumferential WSS distribution can also be utilized, since it can provide more detailed information of WSS variation in anatomical locations. In 
this context, WSS datas were smoothed, i.e. the WSS datas located in every $5^{\circ}$ of the $2 \mathrm{~mm}$ thick band was avaraged, see figure 2. Figures 5 and 7 show contours and circumferential WSS for subject 5, this subject were choosen as an example of the results for selected time points during the cardiac cycle and for both semi-automatic and manual segmentation methods.

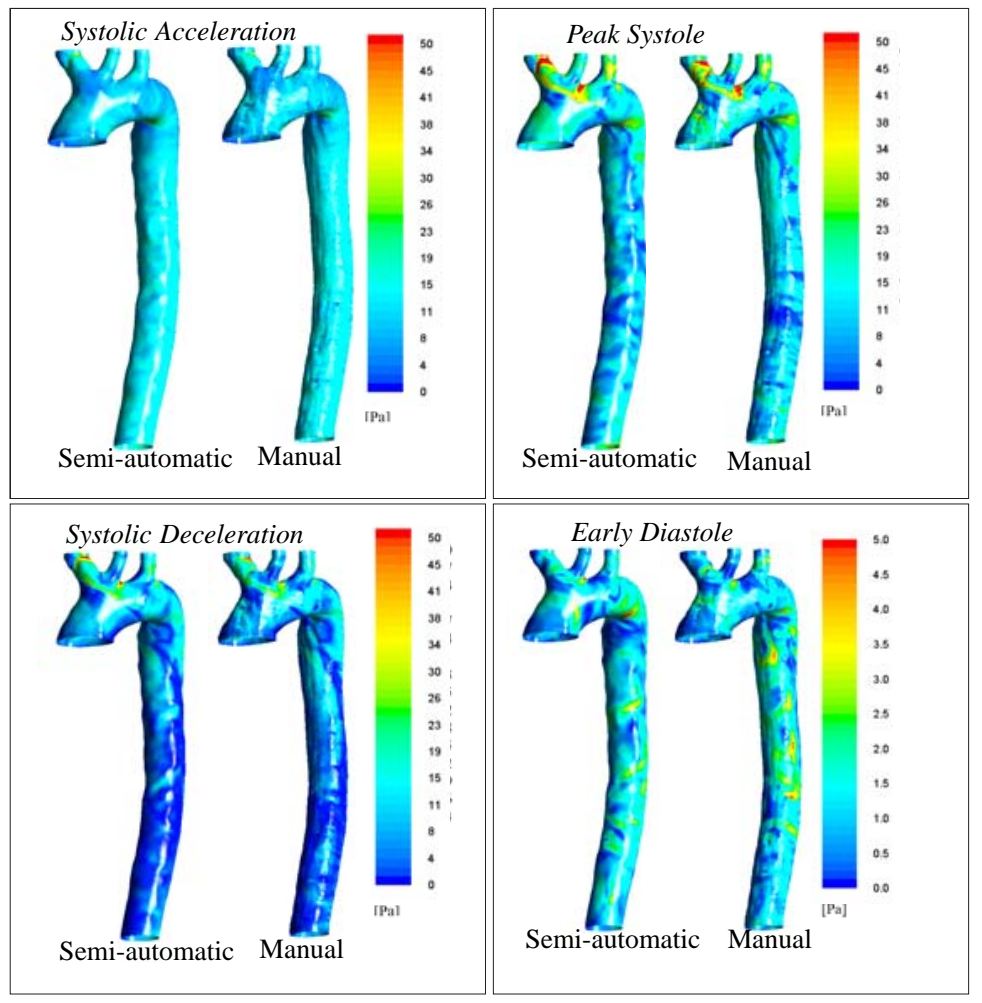

Fig. 5 Contours of WSS for Subject 5, semi-automatic and manual segmentation at Systolic Acceleration, Peak Systole, Systolic Deceleration and Early Diastole. Note the different color-bar scale at Early Diastole.

For comparison of the circumferential WSS results of the two segmentation methods, the Pearson correlation coefficient $r$ has been used. This coefficient gives a single numerical value signifying the level of relation between the two WSS curves, shown in Figure 7. Ta- 


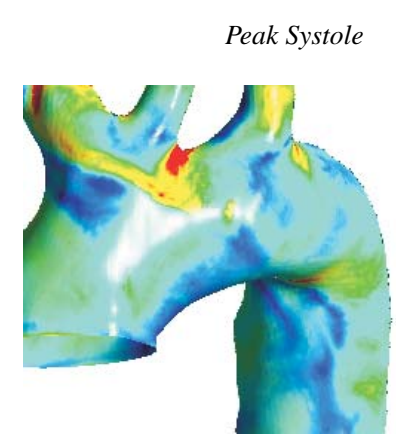

Semi-automatic

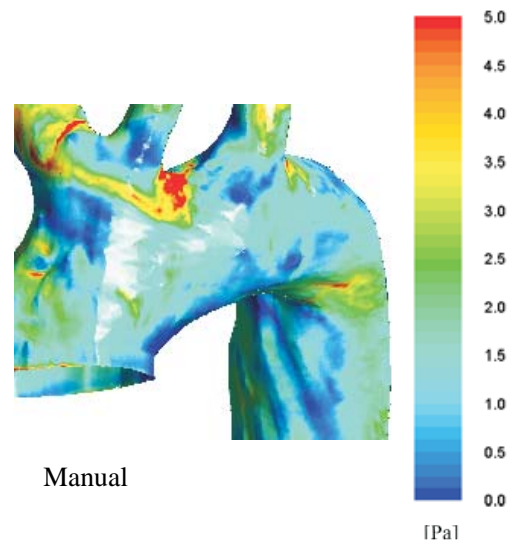

Fig. 6 Zoom in on the aortic arch of WSS contours for Subject 5, semi-automatic (left) and manual segmentation (right) at Peak Systole.
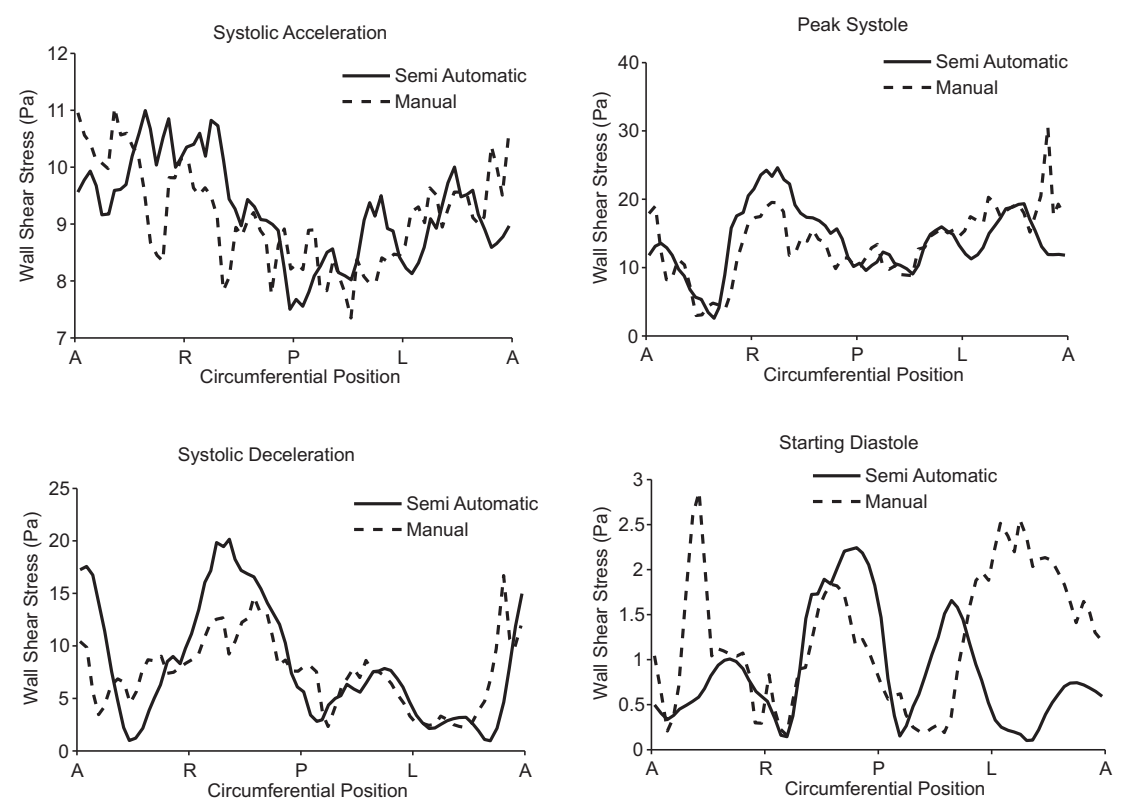

Fig. 7 Circumferential WSS for Subject 5, semi-automatic and manual segmentation methods during Systolic Acceleration, Peak Systole, Systolic Deceleration and Early Diastole. The circumferential positions correspond to: anterior (A), right (R), posterior $(\mathrm{P})$ and left (L).

ble 4 shows the correlation coefficient values between circumferential WSS of each individual within different time points. Positive values close to 1 indicate a strong relation between 
two data sets in an increasing mode and those close to zero represent a weaker relation. The same is valid for a negative correlation coefficient, but in a decreasing fashion. In order to

Table 4 Correlation coefficient (manual vs. semi-automatic segmentation) of every subject for the circumferential WSS.

\begin{tabular}{|c||c|c|c|c|}
\hline Subject & Systolic Acceleration & Peak Systole & Systolic Deceleration & Early Diastole \\
\hline \hline 1 & 0.69 & 0.84 & 0.66 & 0.51 \\
\hline 2 & 0.56 & 0.78 & 0.87 & 0.50 \\
\hline 3 & 0.56 & 0.64 & 0.63 & 0.50 \\
\hline 4 & 0.29 & 0.11 & 0.70 & 0.34 \\
\hline 5 & 0.80 & 0.73 & 0.76 & 0.09 \\
\hline 6 & 0.58 & 0.55 & 0.44 & 0.44 \\
\hline 7 & 0.85 & 0.93 & 0.89 & -0.55 \\
\hline 8 & 0.68 & 0.47 & 0.53 & -0.01 \\
\hline
\end{tabular}

obtain an impression of the general relation between the two segmentation methods, mean circumferential WSS values together with standard deviation can also be utilized. These properties can offer a rather high level of relation between two methods. The mean values are close and the variability or dispersion (standard deviation) of the measured mean values overlap. Plots of mean WSS with their relevant standard deviation around the cross-section for each subject and at four time points are depicted in figure 8. This is useful when viewing the differnces between the subjects and the spatial spread over the cross-section. To fully describe the influence on WSS by the segmentation method, circumferential mean values were constructed for the whole comparison band. The result are shown as Bland-Altman plots in figure 9.

Left part of figure 9 designates general agreement between the segmentation methods, 


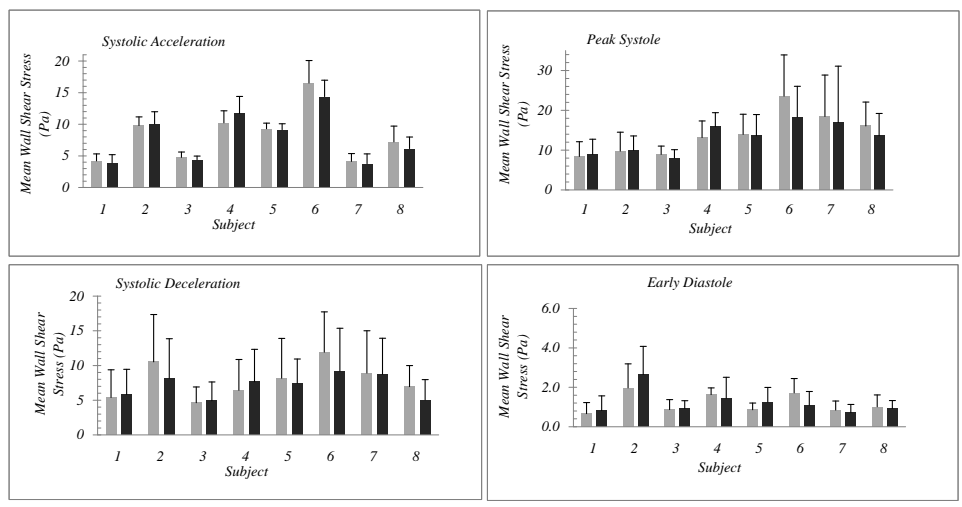

Fig. 8 Circumferential mean WSS and standard deviation at the descending aorta cross-sections, for all subjects, semi-automatic (grey bars) and manual segmentation (black bars) at four time instances of the cardiac cycle: Systolic Acceleration, Peak Systole, Systolic Deceleration and Early Diastole. Note that the scale varies between the figures.

since the plotted points are positioned along the line of equality. The right part of figure 9 is used to investigate any possible relationship between the measurement error and the level of the measurement. The mean difference of the WSS estimations for the four time points is visualized in figure 10 , combined with the inlet volume flow rate for subject 5 . Note the increasing behavior. To clearly distinguish the influence of time point in the WSS results from the different segmentation methods correlation coefficients were also calculated using the circumferential mean WSS values, see table 5. It is important to note the spread in the correlation coefficient.

Table 5 Correlation Coefficient (Manual vs. Semi-Automatic segmentation) of circumferential mean WSS for every subject.

\begin{tabular}{|c|c|c|c|}
\hline \multicolumn{5}{|c|}{ Time Point } \\
\hline Systolic Acceleration & Peak Systole & Systolic Deceleration & Early Diastole \\
\hline \hline 0.99 & 0.96 & 0.88 & 0.68 \\
\hline \hline
\end{tabular}



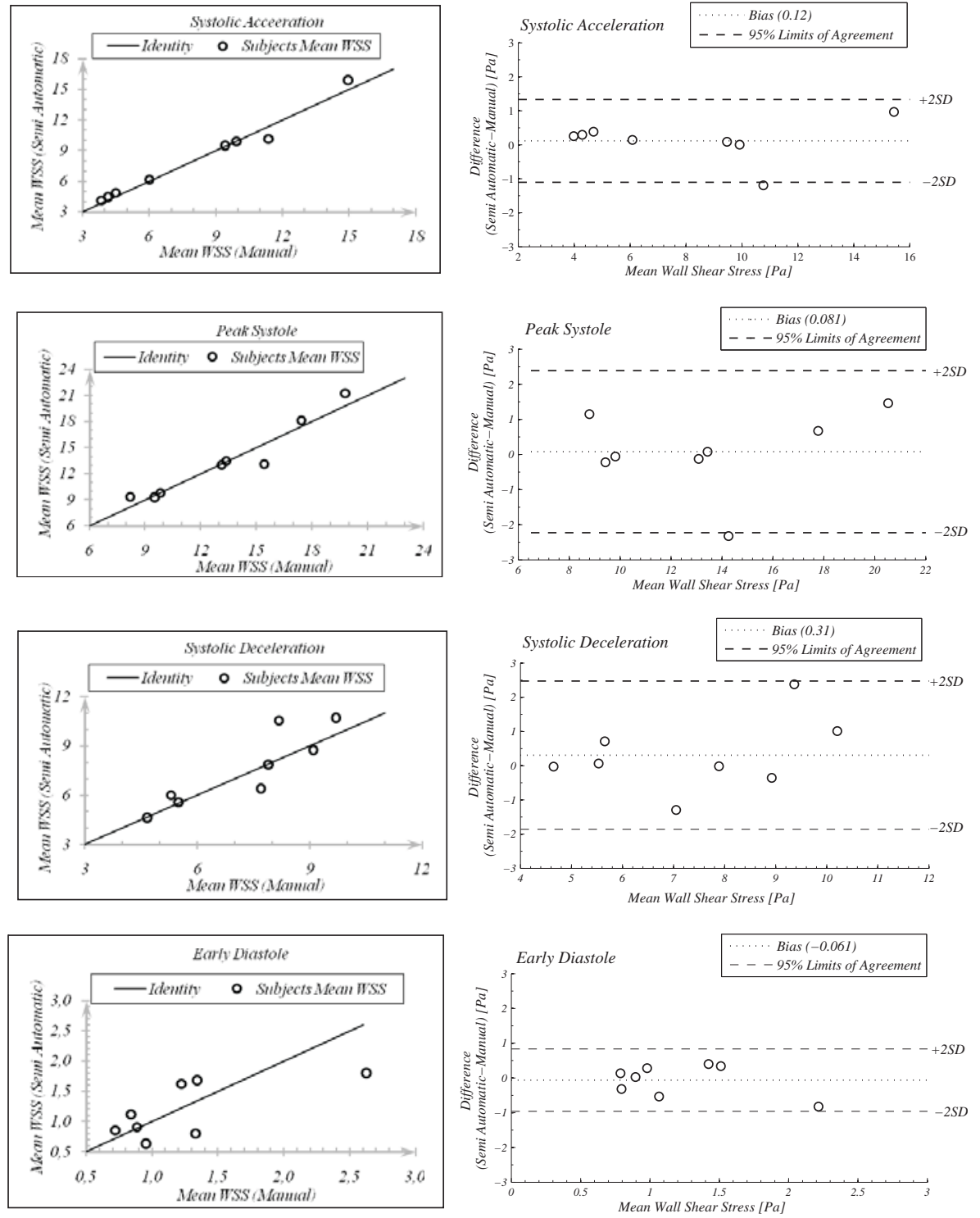

Fig. 9 Left: Circumferential Mean WSS in [Pa] for all subjects, semi-automatic vs. manual during Systolic Acceleration, Peak Systole, Systolic Deceleration and Early Diastole and unity line. Right: Bland-Altman plot with difference in circumferential mean WSS between semi-automatic and manual vs. mean WSS of the two segmentation methods, during Systolic Acceleration, Peak Systole, Systolic Deceleration and Early Diastole. 


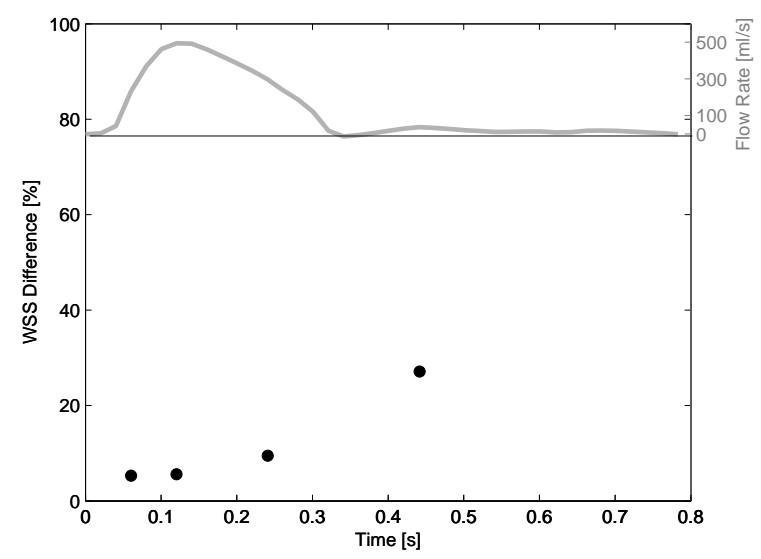

Fig. 10 Mean WSS difference (filled dots, left axis) for all subjects within different time points in the cardiac cycle. Cardiac cycle is represented at the top (grey line, right axis) as the ascending aortic volume flow rate for subject 5 .

\section{Discussion}

In the current study, 3D geometries of the human aorta of 8 healthy volunteers have been reconstructed from magnetic resonance imaging data acquisition by means of both manual and semi-automatic methods. CFD results are compared for all subjects in terms of WSS, both qualitatively and quantitatively. Regarding the geometry of the aortas, the measurement of the LD gives similar results. Furthermore the variability of the LD was low according to figure 4.

As shown in the representative subject 5, (see figurs 5 and 6) the WSS shows signs of good agreement between the semi-automatic and manual segmentation methods. However the surface from the semi-automatic segmentation becomes smoother (figure 5 and 6), which seems more physiologically relevant indicating that the semi-automatic segmentation approach is more appropriate regarding surface smoothness. This way of reasoning lies in line with [22] 
results from the carotid artery. Smoothing can influence the WSS results but with the used protocol this effect seems small as shown in figures 5 and 6, where smoothing for the different segmentation gives different surface smoothness but WSS still is similar. In order to avoid over-smoothing and at the same time reduce the rough inital segmented surface (due to the voxel-based image material) into smooth surface, a wide range of smoothing setting were tested which resulted in an appropriate smoothing setting applicable to all subjects. This consistent settings for all subjects is appropriate to use for this kind of comparision study. Although our experience is that the differneces between smooting setting are rater small, there might be a slightly more optimal approach to the smoothing procedure.

The circumferential distribution of WSS in the ascending aorta was examined and representative results for subject 5 are shown in figure 7. The detailed distribution of WSS agreed well between semi-automatic and manual segmentation. The pooled data from the subjects compares the differences between the curves (e.g. Figure 7) and indicates good agreement between the segmentation methods (e.g. table 4). The agreement between the two segmentation methods is also evident in the left part of figure 9 where it becomes clear that the best agreement is found during the systolic acceleration and peak systole time points of the cardiac cycle.

It is also shown that there are no systematic errors between the two segmentation approaches and the difference bias is generally close to zero, see right part of Figure 9. The agreement becomes smaller in the systolic deceleration and early diastole where the flow is decelerating and relatively slow. This is more clearly shown by the correlation coefficients in table 5 , which generally shows good agreement between the methods, but the level of correlation is lower in the systolic deceleration and even lower in the early diastole. This decreased cor- 
relation level in the early diastole is seen in both table 4 and figure 10. A possible reason might be that the low flow velocities at this point of the cardiac cycle are more sensitive to local geometrical description of the arterial wall. The high WSS resolution presented by our method has not been reported by other groups, which is why further comparison with earlier papers is possible. The relative error in the accuracy and reproducibility findings on the carotid artery by [26] suggests a reported error of $7-10 \%$ for maximum WSS, which is in line with our results for systolic time-positions, where the largest WSS is gained, see figure 10. The temporal variation in the results is new information provided by our study. In the overall view of the WSS for the subjects in figure 8 there are clear differences between the subjects and the deviation between segmentation method is in generally small. The differences in WSS between the subjects is far more prominent compared to influence of the segmentation method.

Hammer et al. [22] compared CFD results from two segmentation approaches similar to ours. They concluded that the semi-automated method had some advantages (e.g. time reduction for segmentation) and disadvantages (e.g. oversmoothing in diseased regions) compared with the manual method. Their work was limited by the number of datasets (only one dataset) and the influence of the time point in the cardiac cycle was not included (steady-state CFD). Finally the comparison was qualitative with limited quantitative evaluation concerning the differences between methods. Our data shows that circumferential averaged WSS in general gives similar results with the two methods, see Figure 8. It is important to note the large differences in WSS between individuals, despite a very homogeneous group of volunteers (see table 1). This type of subject specific WSS results may give valuable information regarding the risk of atherosclerosis development in individual subjects. Averaging WSS results for a whole group of subjects is not recommended, due to the large individual 
variations in WSS.

The results are representative for this particular group of young healthy males only, because both flow and arterial geometry can be influenced by gender, age and cardiovascular disease. One advantage of applying the proposed method on older people is that their vessels in general are more stiff [27], so the assumption of rigid vessel walls is actually more accurate. Regarding patients with aortic constrictions, the method is still valid but turbulence modelling might be needed to capture the flow situation, which mainly will lead to longer simulation time.

\section{Conclusion}

Finally we conclude that the estimated WSS with manual and semi-automatic segmentation methods shows good agreement. In the diastolic part of the cardiac cycle the WSS is more sensitive to small differences in the geometry differences in the geometricy due to the different segmentation methods. Thus, care should be taken when very detailed WSS data is of interest in the human aorta at the diastolic part of the cardiac cycle. The full set of results implicate that the semi-automatic segmentation method can be used in the future as it produces similar WSS results as a manual segmentation approach.

\section{References}

1. P. Libby, P.M. Ridker, G.K. Hansson, Progress and challenges in translating the biology of atherosclerosis, Nature, 473:317-325, 2011

2. A.M. Malek, S.L. Alper, S. Izumo, Hemodynamic shear stress and its role in atherosclerosis. Journal of the American Medical Association, 1;282(21):2035-42, 1999 
3. Q. Long, Y. Xu, B. Ariff, S. A. Thom, A. D. Hughes, A. V. Stanton, Reconstruction of Blood Flow Patterns in a Human Carotid Bifurcation: A Combined CFD and MRI Study, Journal of Magnetic Resonance Imaging, 11:299-311, 2000

4. C. A. Taylor, C. P. Cheng, L. A. Espinosa, B. T. Tang, D. Parker, R. J. Herfkens, In Vivo Quantification of Blood Flow and Wall Shear Stress in the Human Abdominal Aorta During Lower Limb Exercise, Annals of Biomedical Engineering, 30:402-408, 2002

5. A. Leuprecht, S. Kozerke, P. Boesiger, K. Perktold, Blood flow in the human ascending aorta: a combined MRI and CFD study, Journal of Engineering Mathematics, 47:387-404, 2003

6. D. A. Steinman, C. A. Taylor, Flow Imaging and Computing: Large Artery Hemodynamics, Annals of Biomedical Engineering, 33;12:1704-1709, 2005

7. A. D. Augst, B. Ariff, S. A. G. McG. Thom, X. Y. Xu, A. D. Hughes, Analysis of complex flow and the relationship between blood pressure, wall shear stress, and intima-media thickness in the human carotid artery, American Journal of Physiology - Heart and Circulatory Physiology, 293:H1031-H1037, 2007

8. J. Renner, R. Gårdhagen, E. Heiberg, T. Ebbers, T. Länne, M. Karlsson, A Method for Subject Specific Estimation of Aortic Wall Shear Stress, WSEAS Transactions on Biology and Biomedicine, ISSN 1109$9518,3(6): 49-57,2009$

9. R. Erbel, General Cardiology Diseases of the thoracic aorta, Heart, 86:227-234, 2001.

10. D. Steinman, Image-based computational fluid dynamics modeling in realistic arterial geometries, Annals of Biomedical Engineering, 30:483-497, 2002

11. J. Suo, J. Oshinski, D.P. Giddens, Effects of wall motion and complience on flow pattern in the ascending aorta, Jornal of Biomechanical Engineering, 125:347-354, 2003

12. D. Mori, T. Yamaguchi, Computational Fluid Dynamics Modeling and Analysis of the Effect of 3-D Distortion of the Human Aortic Arch, Computer Methods in Biomechanics and Biomedical Engineering, $5: 249-260,2002$

13. L Morris, P Delassus, A Callanan, M Walsh, F Wallis, P Grace, and T McGloughlin. 3-D numerical simulation of blood flow through models of the human aorta. Journal of Biomechanical Engineering, 127(5):767-775, 2005.

14. J.G. Myers, J.A. Moore, M. Ojha, K.W. Johnston, C.R. Ethier, Factors Influencing Blood Flow Patterns in the Human Right Coronary Artery. Annals of Biomedical Engineering, 29:109-120, 2001 
15. E. Heiberg, L. Wigström, M. Carlsson, A. Bolger, M. Karlsson, Time resolved three-dimensional automated segmentation of the left ventricle, In: Computers in Cardiology, Lyon, France, 32: 599-602, 2005 http://segment.heiberg.se.

16. J.A. Sethian, Level set methods and fast marching methods, Cambridge University Press, Cambridge, USA, 1999

17. J.M. Bland, D.G. Altman, Statistical methods for assessing agreement between two methods of clinical measurement, Lancet, 8;1(8476):307-310, 1986

18. J.L. Rodgers, W.A. Nicewander, Thirteen Ways to Look at the Correlation Coefficient, The American Statistican, 42;1:59-66, 1988

19. D.A. Steinman, D.A. Vorp, C.R. Ethier, Computational modeling of arterial biomechanics: Insights into pathogenesis and treatment of vascular disease, Journal of Vascular Surgery, 2003;37:1118-1128.

20. C.A. Taylor, M.T. Draney, Experimental and computational methods in cardiovascular fluid mechanics, Annual Review of Fluid Mechanics, 2004;36: 197-231.

21. K.W. Lee, N.B. Wood, X.Y. Xu, Ultrasound image-based computer model of a common carotid artery with a plaque Medical Engineering and Physics, 2004;26: 823-840.

22. S. Hammer, A. Jeays, P.A. Allan, R. Hose, D. Barber, W.J. Easson, P.R. Hoskins, Acquisition of 3D Arterial Geometries and Integration with Computational Fluid Dynamics, Journal of Ultrasound in Medicine and Biology, Vol. 35, No. 35, N0. 12, pp. 2069-2083, 2009.

23. C. Y. Wen, A. S. Yang, L. Y. Tseng, and J. W. Chai. Investigation of pulsatile flowfield in healthy thoracic aorta models, Annals of Biomedical Engineering, 38(2):391-402, 2010.

24. C. Cheng, D. Tempel, R. van Haperen, A. van der Baan, F. Grosveld, M. J. Daemen, R. Krams, and R. de Crom, Atherosclerotic lesion size and vulnerability are determined by patterns of fluid shear stress, Circulation, 113:2744-2753, 2006

25. F. J. Gijsen, J. J. Wentzel, A. Thury, B. Lamers, J. C. Schuurbiers, P. W. Serruys, and A. F. van der Steen, A new imaging technique to study 3-D plaque and shear stress distribution in human coronary artery bifurcations in vivo Journal of Biomechanics, 40:2349-2357, 2007.

26. A. D. August, D. C. Barrat, A. D. Hughes, F. P. Glor, S. A. McG. Thom, and X. Y. Xu, Accuracy and reproducibility of CFD predicted wall shear stress using 3D ultrasound images, Journal of Biomechanical Engineering, 125:218-222, 2003. 
27. T. Länne, H. Stale, H. Bengtsson, D. Gustafsson, D. Bergqvist, B. Sonesson, H. Lecerof, and P. Dahl, Noninvasive measurement of diameter changes in the distal abdominal aorta in man, Ultrasound in Medicine and Biology, 18:451-457, 1992.

28. J. Lotz, C. Meier A. Leppert, and M. Galanski, Cardiovascular Flow Measurement with Phase-Contrast MR Imaging: Basic Facts and Implementation. Radiographics, 22(3):651-671, 2002. 